\title{
Clinical Laboratory Medicine as the Emerging Healthcare Partner
}

\author{
Martin H. Bluth, MD, PhD* \\ Department of Pathology, Wayne State University School of Medicine, Detroit; \\ Consolidated Laboratory Management Systems, Southfield; Michigan Surgical Hospital, \\ Warren, Michigan, USA
}

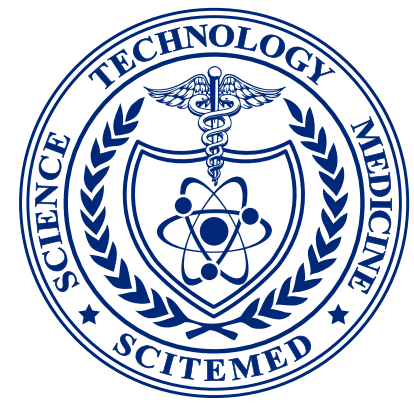

$\mathrm{T}$ he landscape of Medicine is ever changing. Improvements in healthcare continue to mature, and various clinical disciplines continue to evolve. The discipline of Clinical Pathology and Laboratory Medicine, also referred to as "fluids medicine" continues to evolve as well. Biomarkers for disease diagnosis, prognosis and maintenance have provided a wellspring of opportunity for the clinician and layperson alike. Select blood tests have honed in on certain disease spaces that are more sensitive and specific for ailments than previous iterations. In the case of heart disease, one can follow the maturation of lactate dehydrogenase to myoglobin to creatinine kinase MB fraction and most recently troponins as a proof of principle to how laboratory medicine keeps pace as well as directs disease diagnosis and management.

Genetic testing has fostered an explosion of prognostication and predictive value of disease which not only affects the index case but also his/her progeny. Such genetic results can affect the decision of whom to marry as well as the decision to abort. Various glitterati have taken up cause, providing considerable national and global visibility to the rubric of laboratory medicine. Actress Angelina Jolie has fostered a unique visibility for breast cancer [1], the late pop-star Prince has had requests for paternity testing for claims to his estate [2], and various blood testing segments have been featured on the Oprah network and related subsidiaries [3].

Furthermore, what has previously been in the domain of the health care provider with respect to the ordering of blood testing has expanded to the Direct to Consumer (DTC) marketplace. The laboratory has provided the backbone for various emerging industries that have become timely buzzwords for health including "wellness" panels which can be customized for the athlete, as well as tests for those concerned with sexually transmitted diseases, allergic disease, and diabetes to name a few. Anyone can walk into a drug store and test for a variety of items including human immunodeficiency virus, urinary tract infection, and drugs of abuse among others. Personal discretion has precipitated this market space to some degree where many individuals prefer to monitor their health independently and seek medical attention when they feel such is required.

To this end, laboratories have provided direct lab access to consumers, with ease of specimen procurement, via cheek swab testing as well as blood or other body fluids that can be obtained in the comfort of one's own home or at a participating in-pharmacy phlebotomy stations for subsequent direct ship to the processing laboratory [4]. Additional impetuses for the DTC revolution stems from various shifting of ideals. These range anywhere from ease of access, control of personal health, reduced time and costs by obviating the doctor's office visit, keeping off the insurance carrier grid, avoiding being labeled with "pre-existing illness" in a chart which often follows the patient in perpetuity, and better cost centers with cash pay than insurance covered copay, among others. This DTC approach has had mixed reviews and accessibility can vary from state to state [5].

Lab testing has also become intrinsic to corporate, government, legislative and, in certain cases, punitive reform. The discipline of toxicology within the rubric of clinical chemistry has been used to dismiss patients from their care providers due to resulting positive drug screens where the clinical practice has a zero drug tolerance policy and forensic testing can be used to provide support for incarceration or job dismissal when chain of custody processes are employed [6]. Various political pundits utilize lab testing to maintain their steadfast positioning where it would service select initiatives. Certain establishments provide incentives for maintaining ideal glucose and cholesterol blood levels in addition to weight and exercise regimens as a mean to reduce health care costs which can be in the form of tax rebates or other credits [7].

Although the clinical laboratory testing approach serves to provide ancillary diagnostic and prognostic support to the health care provider in line with other modalities (history, physical exam, imaging, etc.), it has arguably metamorphosized to become the "medical gatekeeper" in certain settings. It is unusual to have someone present to the emergency room with a suspected diagnosis of acute abdomen who is not subjected to a battery of blood tests to aid in the differential diagnosis of whether the case is medical or surgical in nature. Part of the reason for this is that lab testing has become easily accessible, miniaturized, rapid, economical, and ostensibly easy to interpret. Many emergency rooms and acute care centers have on-site point of care (POC) instruments which can determine blood counts, chemistries, electrolytes and the like in moments with minimal operator requirements.

To this end, the adaptation of micro-electronic mechanical sensors (MEMS) has catapulted this ease of use and adaptability to mainstream medicine. For example, whereas blood potassium concentration used to be determined by flame photometry and interpreted by a clinical pathologist, it is now assessed by ion selective electrodes such as valinomycin and results in seconds by automated instrument that can be operated by a non-laboratorian, often in a POC setting. MEMS has also expanded testing opportunities by adapting other industries to laboratory medicine such as the marriage of Coulter counter technology with dot-matrix printing to birth flow cytometry which has become integral to the field of hematopathology and blood cancer diagnosis and management.

Part of the reason for robust lab testing also stems from inflated litigation awards for malpractice suits which have caused doctors and hospitals to implement a multitude of interventions including lab tests to mitigate the risk of 'missing something' while being mindful of cost containment [8]. To this end, clinical pathology expert witness and testimonials are not only used in the tried and true arena of forensics made famous by television programs such as Quincy, Crime Scene Investigation, Bones and the like, rather, they are also being solicited for laboratory test selection logic, timeliness of transfusion, medical record review, and specimen processing, among other things, which can be integral to adjudicating wrongful death suits as well as other cases of morbidity and mortality.

On the surface it seems ideal to avail widespread use of the clinical laboratory in ways which were never available 40 years ago. However, there is a darker side to this, one that is more insidious than intentional but has pervaded the medical machine to foster misuse of lab tests. Certain healthcare providers will use lab test results as a "light switch"-a sort of absolutism type of 'yes or no' gestalt-to incorporate into their patient management to vindicate or vilify based on one test result. Examples of such can include a 47-year-old male dock worker fired from his job as a result of a positive drug screen; a 38-year-old mother of three having her 
insurance coverage affected due to a newly identified gene carrier status; a 7 year old child placed in foster home as a result of a positive lead testing result. Such hasty sequelae may have resulted from a number of impo-sitions that have encroached on the medical marketplace. Compared with decades past, doctors have an average of 8 minutes to see their patient [9], malpractice insurance as a result of inflated wrongful practice awards continues to climb, payor mix fee schedules continue to tighten, and the promise of the new and improved 'best test of the day to identify all the patient ills' continues to confuse and confound providers as well as their patients [10].

Lab testing is big business. Test results find their way into actuarial tables used for life insurance premiums, health insurers set and reset utilization standards and justification logic to determine if a test is covered and cost containment is ever present. For example, in certain cases, a physician may need to explain to a Hashimoto's thyroiditis patient that the insurance company will not pay for T4 or anti-thyroid antibody testing if the TSH is "normal". Furthermore, in an effort to economize and streamline care, in certain cases the matrimony of doctors, patients, and ancillary services (laboratory, imaging, etc.) have coalesced into various versions of a network based providership (Patient Centered Medical Homes, Clinical Integration Networks, Accountable Care Organizations, etc.) In such cases, if the patient is "in network" then he/she is covered to the highest percentage of base (less copay and deductible in certain cases) but if one uses a non-participating provider he/she is balanced billed often for a higher non-network rate [11].

There is a new concern as well. There has been an impetuous initiative to have the laboratory increase its position of "medical gatekeeper" by superseding the primary care physician's management decisions and justifying the utilization of physician ordered labs. Such an imposition could further unravel the fabric of healthcare by eroding trust between physician and laboratory, similar to the erosion experienced between physician and patient in certain respects [12]. For example, it is not prudent to position the laboratory as the "sodium and potassium police" as a post hoc initiative to address suspected physician lab ordering misuse for the sole intent of cost mitigation. This initiative has likely resulted from a frantic need to curtail spending without the sensitivity and integration involved in the doctor - patient - laboratory amalgam. Further, the legalities of such an initiative are questionable as the management of the patient rests with the primary caregiver who moderates the total care rather than an ancillary support system (lab, imaging, pharmacy) which relates to a particular aspect of the comprehensive care cycle, and also needs to be mindful of perceived incentivization concerns [13].

However, there is a way to remedy, normalize and effectively utilize laboratory medicine in the ever-changing medical marketplace. The clinical laboratory is an integral partner in healthcare. Its position in the medical armamentarium is as much to provide ancillary support to the history and physical-the mainstay of the physician patient relationship-as it is to provide guidance on forecasting for patient management, all under the transparent rubric of clinical application. The understanding of the limitations of the laboratory, the interfering factors that can confound and subsequently explain a curious test result, and the differences in technology employed by one lab over another are all unique to the practice of laboratory medicine and are not always appreciated by the general medical practitioner [14].

The clinical pathologist/laboratorian is also key to providing logic on the economics related to testing that renders ancillary vs diagnostic information for patient management. The tempering of a given test result with the human being to whom it belongs, to mitigate the worry of over BRCA interpretation (genetics), dismissal from an addiction clinic for positive cocaine on urine testing (toxicology), differential diagnosis of multiple myeloma (serum protein electrophoresis), infectious disease determination (serology vs. nucleic acid testing), blood product/stem cell transfusion compatibility (immunohematology, histocompatibility), pre to post analytic laboratory test resulting (laboratory informatics, automation), and the like all lie within the wheelhouse and expertise of the laboratorian. To this end, recent pathology-centric consultation programs have been launched to provide direct interaction with the patient on pathology related issues [15] and clinical pathology telehealth programs have been shown to improve outcomes for subspecialty referral patterns in certain health systems [16].

The clinical pathologist/laboratory medicine physician serves as the ideal health care partner to synergize with the patient-physician-unit to temper curious results, obviate the "light switch" effect, provide guidance on current and emerging test utilization and management, navigate the DTC marketplace and educate patient and physician alike as required, and to ultimately incorporate lab test results into actual optimization of patient care. It is through this approach that the patient-physician-laboratorian triumvirate can maximize the experience to positively affect patient centric, evidenced based, fiscally responsible healthcare in the ever-changing medical landscape.

\section{Article Information}

*Correspondence: Martin H. Bluth, MD, PhD

Department of Pathology, Wayne State University School of Medicine, Detroit; Consolidated Laboratory Management Systems, Southfield; Michigan Surgical Hospital, Warren, Michigan, USA.

Emails: toxdocs@gmail.com; mbluth@med.wayne.edu

Received: Aug. 28, 2017; Accepted: Aug. 31, 2017; Published: Sep. 14, 2017

DOI: 10.24983/scitemed.jacp.2017.00031

Copyright (c) 2017 The Author. This is an open-access article distributed under the terms of the Creative Commons Attribution 4.0 International License (CC-BY).

\section{Funding: None}

\section{Conflict of Interest Disclosures: None}

\section{References}

1. Celebrities and Genetic Testing 2015. Available at: http://www. genengnews.com/gen-polls/celebrities-and-genetic-testing/296

2. Prince and Celebrity Paternity Testing 2016. Available at: https:// dna- testing.com/prince-and-celebrity-paternity-testing/

3. 5 Blood Tests That Are Worth the Prick. Available at: http://www. op- rah.com/health/the-most-important-blood-tests

4. Direct-to-Consumer Laboratory Testing. American Association for Clinical Chemistry 2015. Available at: https://www.aacc.org/ health-and-science-policy/advocacy/position-statements/2015/direct-to-consumer-laboratory-testing

5. O'Conner A. Direct-to-Consumer Lab Tests, No Doctor Visit Required 2016. Available at: https://mobile.nytimes.com/blogs/well/2016/06/ 06/direct-to-consumer-lab-tests-no-doctor-visit-required/

6. What Employees Need to Know About DOT Drug \& Alcohol Testing 2014. Available at: https://www.transportation.gov/odapc/employee-handbook-english

7. Memorandum. Available at: https://www.irs.gov/pub/irswd/2016220 31.pdf

8. Jena $A B$, Schoemaker L, Bhattacharya J, Seabury SA. Physician spending and subsequent risk of malpractice claims: observational study. BMJ 2015;351:h5516.

9. Chen PW. For new doctors, 8 minutes per patient. New York Times 2013. Available at: http://well.blogs.nytimes.com/2013/05/30/fornew-doctors-8-minutes-perpatient/

10. LaBeau KM, Simon M, Steindel SJ. Quality control of test systems waived by the Clinical Laboratory Improvement Amendments of 1988. Perceptions and practices. Archives of Pathology \& Laboratory Medicine 2000;124:1122-1127.

11. Anne Paxton. Clampdowns on out-of-network billing climb 2016. Available at: http://www.captodayonline.com/clampdowns-network-billing-climb/ 


\section{EDITORIAL}

12. Hawley K. Trust and distrust between patient and doctor. Journal of Evaluation in Clinical Practice 2015;21:798-801.

13. Emi Tabb. The Challenges of the Practitioner-Laboratory Relationship 2016. Available at: https://blog.hint.com/the-challenges-of-the-practioner-laboratory-relationship

14. Hickner J, Thompson PJ, Wilkinson T, et al. Primary Care Physicians' Challenges in Ordering Clinical Laboratory Tests and Interpreting Results. Journal of the American Board of Family Medicine 2014;27:268-274.
15. Amy Carpenter Aquino. New pathology patient consult program takes off 2017. Available at: http://www.captodayonline.com/ new-pathology-patient-consult-program-takes/

16. Risin SA, Chang BN, Welsh KJ, et al. Exploring New Ways to Deliver Va-lue to Healthcare Organizations: Algorithmic Testing, Data Integration, and Diagnostic E-consult Service. Annals of Clinical \& Laboratory Science 2015;45:239-247. 